\title{
F4E R\&D PROGRAMME and RESULTS on in-VESSEL DUST and TRITIUM
}

\author{
F. Le Guern ${ }^{a^{*}}$, W. Gulden ${ }^{\mathrm{a}}$, S. Ciattaglia ${ }^{\mathrm{b}}$, G. Counsell ${ }^{\mathrm{a}}$, A.Bengaouer ${ }^{\mathrm{c}}$, J.Brinster ${ }^{\mathrm{c}}$, F.Dabbene $^{\mathrm{c}}$, A.

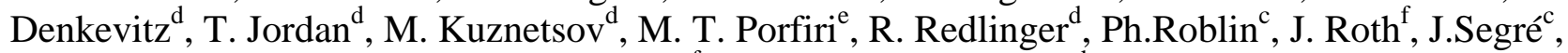 \\ K. Sugiyama ${ }^{\mathrm{f}}$, I. Tkatschenko ${ }^{\mathrm{c}}, \mathrm{Z}$. Xu ${ }^{\mathrm{d}}$
}

\author{
${ }^{a}$ Fusion for Energy Joint Undertaking, Josep Pla 2, Torres Diagonal Litoral - B3, 08019 Barcelona, Spain \\ ${ }^{b}$ ITER organization, CS 90 046, 13067 St Paul lez Durance, France \\ ${ }^{c}$ CEA:, DEN, DM2S, SFME F-91191 Gif-sur-Yvette, France. \\ ${ }^{d}$ KIT: Institut für Kern- und Energietechnik 76344 Eggenstein-Leopoldshafen, Germany \\ ${ }^{e}$ ENEA: FPN-FUS-TEC (Fusion Safety \& Environment), Via Enrico Fermi, 45, 00044 Frascati, Italy \\ fIPP: Materials Research Division,Max-Planck-Institut für Plasmaphysik, D-85748 Garching, Germany
}

\begin{abstract}
In a Tokamak vacuum vessel, plasma-wall interactions can result in production of radioactive dust and $\mathrm{H}$ isotopes (including tritium) can be trapped both in in-vessel material and in dust. The vacuum vessel represents the most important confinement barrier to this radioactive material. In the event of an accident involving ingress of steam to the vacuum vessel, hydrogen could be produced by chemical reactions with hot metal and dust. Hydrogen isotopes could also be desorbed from in-vessel components, e.g. cryopumps. In events where an ingress of air to the vacuum vessel occurs, reaction of the air with hydrogen and/or dust therefore cannot be completely excluded. Due to the radiological risks highlighted by the safety evaluation studies for ITER in normal conditions (e.g. in-vessel maintenance chronic release) and accidental ones (e.g. challenge of vacuum vessel tightness in the event of a hydrogen/dust explosion with air), limitations on the accumulation of dust and tritium in the vacuum vessel are imposed as well as controls over the maximum extent of the quantity of accidental air ingress. ITER IO has defined a strategy for the control of in-vessel dust and tritium inventories below the safety limits based primarily on the measurement and removal of dust and tritium. In this context, this paper will report on the efforts under F4E responsibility aimed at developing a number of the new ITER baseline systems. In particular this paper, after a review of safety constraints and ITER strategy, provides the status of:1) Tasks being launched on diagnostics for in-vessel dust inventory measurement 2) Experiments to enrich the data about the effectiveness of desorption of tritium from Be at $350^{\circ} \mathrm{C}$ (divertor baking aiming to release significant amount of tritium trapped in Be co-deposit 3) On-going R\&D programme (experimental and numerical simulation) at FZK, CEA and ENEA on in-vacuum vessel $\mathrm{H} 2$ dust explosion.
\end{abstract}

\section{Introduction}

In a Tokamak Vacuum Vessel (VV), Plasma Wall Interaction phenomena induce tritium trapping and dust production in the Vacuum Vessel. Due to radiological and explosion risks pointed out by safety evaluation studies, the accumulation of dust and tritium in the VV could significantly impact the operation of ITER. In view of ITER licensing and operation, it is needed to improve the knowledge and to develop and assess techniques allowing the monitoring of dust and tritium inventories in the $\mathrm{VV}$ and also mitigation techniques allowing detritiation, dust removal and explosion mitigation. Such techniques are also needed on a longer perspective for future fusion reactors such as DEMO.

This paper includes three main parts: In the first part, safety constraints with respect to in-vessel dust and tritium issues on ITER are outlined. In the second part, ITER strategy is summarized. In the third part, the programme developed in F4E in support to ITER strategy is detailed and some results are provided.

\section{Safety constraints on ITER}

In order to comply with the French nuclear regulatory authority rules safety analysis studies have been carried out [1]. Reference accident scenarios have been justified, source term calculation performed and safety limits with regards to dust quantity and tritium inventory defined as follows:

- $1000 \mathrm{Kg}$ of dusts in the vacuum vessel (VV)

- $1000 \mathrm{~g}$ of tritium in the VV (including the cryo-pump inventory)

Moreover, safety studies [2] have shown that in case of in VV coolant leakage (double end pipe break) followed by steam/air ingress, dust located on hot surfaces (mainly on the divertor region), could produce significant amounts of $\mathrm{H}_{2}$ which could induce an explosion. The calculations evaluated that the latter could generate a transient peak pressure above the VV design pressure. In order to mitigate this risk the studies recommended to limit the amount of dusts deposited on 
hot divertor surfaces (PFCs surface) to the following values: $11 \mathrm{Kg}$ of Be alone or $76 \mathrm{Kg}$ of $\mathrm{W}$ alone.

\section{ITER strategy}

Considering in-vessel dust and tritium issues, two main priorities are defined in ITER strategy:

- the first one consists in keeping dust and tritium inventories below safety limits. Therefore it is needed to develop and assess a set of measurement techniques together with efficient "house-keeping" techniques;

- furthermore, in order to cope with uncertainties on inventory determination, the second priority puts the emphasis on H2/dust explosion prediction (code development and validation) and mitigation method development.

\subsection{In-VV Dust inventory}

The current ITER assumptions [4] regarding dust production are based on feed-back experience and expertise acquired on existing tokamaks. The following assumptions are considered in terms of dust production:

- $\quad$ steady state + ELMs $\left(<0.5 \mathrm{MJ} / \mathrm{m}^{2}\right): 75$ g/discharge

- $\quad$ plasma disruption: $5 \mathrm{~kg} /$ major disruption

With these assumptions, the dust and tritium inventory control is congruent with the planned replacement of divertor cassettes. The progressive starting of operation of ITER will allow the full validation of the assumptions made.

It is foreseen[4] to:1) remove dust at any planned and unplanned in-vessel intervention via Remote Handling (particularly the Multi Purpose Deployer, recently introduced into ITER baseline). With the divertor cassettes in, the access to the areas where dust should be accumulated will be limited. When the cassettes will be replaced the cleaning will be almost total.

Evaluation of dust inventory will be performed through erosion/deposition measurements (In-Vessel Viewing System, Divertor Erosion Monitor) and local dust measurement techniques (Capacitive Diaphragm Monitor and removable samples).

\subsection{In-VV Tritium inventory}

Fuel retention studies are being carried out in many tokomaks to improve understanding of the processes and to allow better predictions for the tritium wall inventory in ITER. Two complementary types of methods are used to determine the fuel retention: 1) Gas balance (difference between the fuel quantity injected and recovered, typically for few discharges or a full day of operation with long term data from Tore Supra). 2) Post mortem tile analysis (analysis of removed tiles and samples, deposition monitors, rotating collector) supported by in situ local measurements such as quartz micro-balance and laser-based methods.
Hydrogenic species are either trapped in co-deposited layers on top of plasma facing components, in dusts or in the bulk of the wall material (implantation-diffusion).

Estimations [3], based on databases (wall fluxes, erosion/deposition rates, saturation level of fuel versus material $(\mathrm{W}, \mathrm{C}, \mathrm{Be})$ and code calculations (D+T fluxes, transport codes ) show that the safety tritium limit could be reached after a few thousand discharges (for the material mix W, Be mix foreseen for D-T operation on ITER).

The strategy [4] foreseen for maintaining the tritium inventory below the safety limit is to Detritiate (release of tritium trapped in Be co-deposit) by divertor baking at $350{ }^{\circ} \mathrm{C}$ when necessary a few times a year (see fig. 1 ).

Furthermore it is also foreseen to implement optimized wall conditioning techniques together with $\mathrm{RH}$ detritiation techniques (photonic methods) and to proceed with dust and co-deposited layer removal (in which the most significant amounts of tritium is trapped).

Evaluation of tritium inventory will be achieved by gas balance and by analysis of material removed from the VV. It is also foreseen to use a RH laser-based technique (Laser Induced Breakdown Spectroscopy) to evaluate distribution of tritium within the machine.

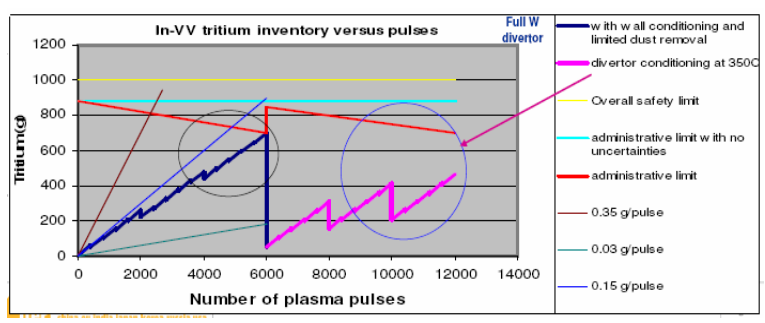

Fig. 1: in-VV tritium inventory versus plasma pulses

\subsection{H2/Dust explosion modelling and mitigation methods}

ITER strategy is based on the development and validation of a $3 \mathrm{D}$ numerical tool able to calculate radiological release consequently to an in-vessel H2/Dust explosion.

This 3D tool and the experimental programme shall in particular address the possibility of implementing mitigation methods such as inert gas injection and igniters.

\section{F4E R\&D Programme}

F4E has been awarded by ITER IO with ITER Task Agreements in order to perform R\&D activities on dust inventory measurement systems, on the divertor baking method and on hydrogen/dust explosion.

\subsection{Dust inventory diagnostics}

F4E is launching three Grants with Associations in Europe to perform feasibility studies on:1) Divertor Erosion Monitor (DEM) 2) Capacitive Diaphragm Monitor (CDM) 3) Hot Dust Measurement using Steam Injection. 


\subsubsection{Divertor Erosion Monitor}

Erosion of material from the divertor targets in ITER will represent one of the largest sources of dust in the vessel. Measurement of the net erosion (i.e. erosion and local redeposition), will provide a useful indicator for the total in-vessel dust inventory. Consequently a DEM system has been recently included into the ITER baseline, subject to satisfactory evaluation of its performance. The objective is, using an optical technique, to allow the measurement of material erosion and deposition occurring on vertical targets (see fig. 2) of the ITER divertor. This task is to evaluate the technical feasibility of making this measurement; identify and select technical options; demonstrate that integration of a DEM is practical and estimate system development costs following a project oriented approach.

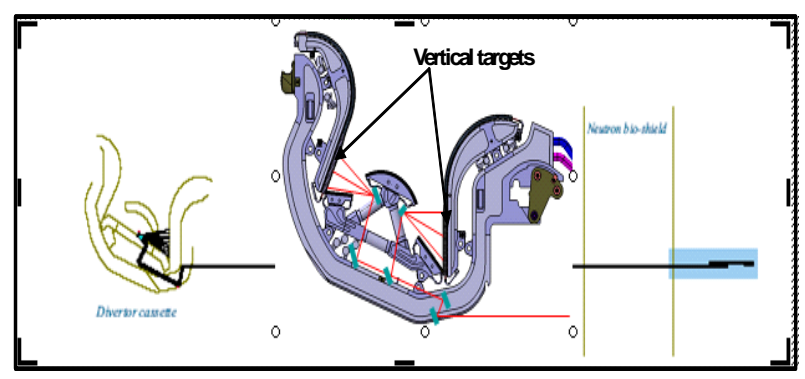

Fig. 2: Divertor Erosion Monitoring sytem (pre-conceptual design)

\subsubsection{Capacitive Diaphragm Monitor}

Previous studies have indicated that the only practical approach to measuring this quantity is by extrapolation from a large number of local measurements. The best candidate sensor for the ITER environment appears to be a CDM [4] and an array of such sensors has been recently included into the ITER baseline subject to satisfactory evaluation. The CDM relies on measuring the change in capacitance between a thin diaphragm and a reference electrode (see fig. 3) as the diaphragm distorts under the weight of dust accumulated on its surface. This task is to evaluate the technical feasibility of making this measurement; demonstrate that integration of a CDM is practical and estimate system development costs following a project oriented approach.

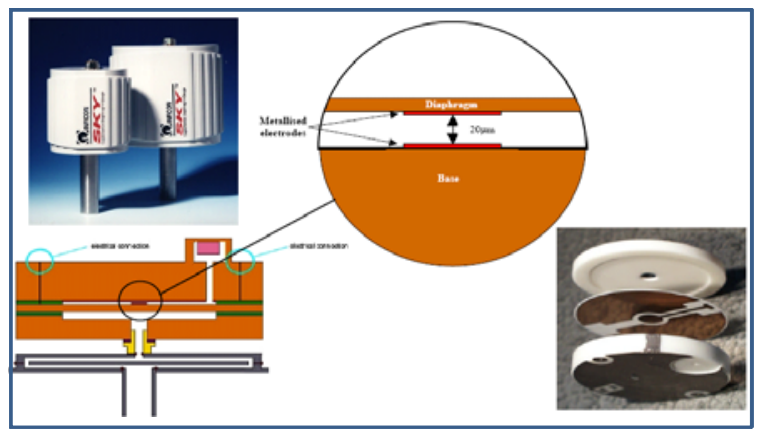

Fig. 3: Capacitive Diaphragm Monitor

\subsubsection{Hot dust measurement using water vapour injection}

A conceptually simple and direct way [5] to evaluate the likely hydrogen production is to intentionally introduce a known, small quantity of water vapour into the V.V. and measure the hydrogen produced, allowing extrapolation to the water vapour ingress expected in a LOCA event.

The task being launched at F4E is to evaluate the technical feasibility of making this measurement. The goal is to perform experiments in a small scale set-up (Vacuum Vessel) allowing water steam injection and chemical reaction with the "hot dust", dust temperature control and hydrogen production measurement.

\subsection{Divertor Baking}

ITER strategy is based on the assumption that divertor baking at $350^{\circ} \mathrm{C}$ allows a $50 \%$ release of tritium trapped.

It has been therefore decided to proceed with an experimental programme to enhance the available database. This programme has been launched in IPP and consists of: 1) preparing realistic layers (Be, Be2C and Be12W) deposited on variable substrates (C, Be and W) and proceeding with $\mathrm{D}$ implantation 2) characterizing $\mathrm{D}$ content by Nuclear Reaction Analysis 3) analyse outgasing ( $\mathrm{T}$ increase) by Thermal Desorption Spectroscopy.

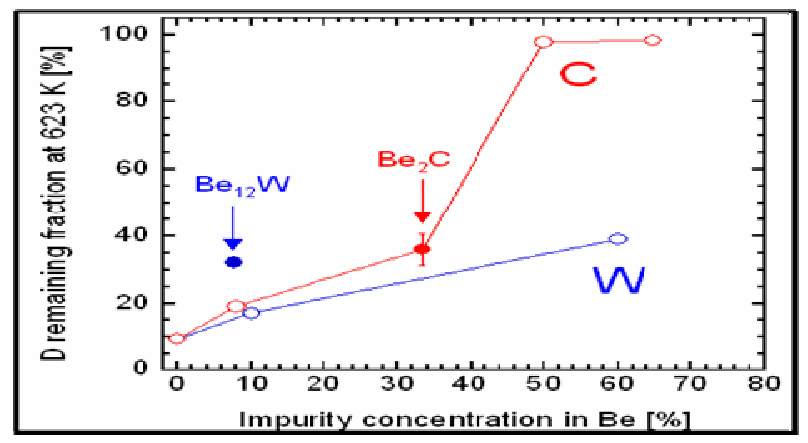

Fig. 4: D remaining fraction at $623 \mathrm{~K}$ in Be-containing samples (D implantation at room temperature) as a function of each impurity ( $\mathrm{W}$ or $\mathrm{C}$ ) concentration in $\mathrm{Be}$

Experimental results[6] indicate (see fig. 4) that the assumption of a $50 \%$ release of tritium is realistic except for deposits rich in C. But, since it is foreseen for the nuclear phase (DT) to operate ITER with a full W divertor, this assumption seems relevant.

\subsection{H2/Dust explosion: Code development and validation experimental programme}

The goal of the R\&D programme on H2/Dust explosion is to develop and validate a $3 \mathrm{D}$ numerical tool able to model H2/Dust explosions in realistic conditions. In particular, this tool shall allow determination of: 1) combustible mixture generation 2) combustion process 3) mechanical and thermal loads on the vessel and consequences. The programme (see fig. 5) aims at carrying out in parallel code development activities and necessary experiments for validation. 


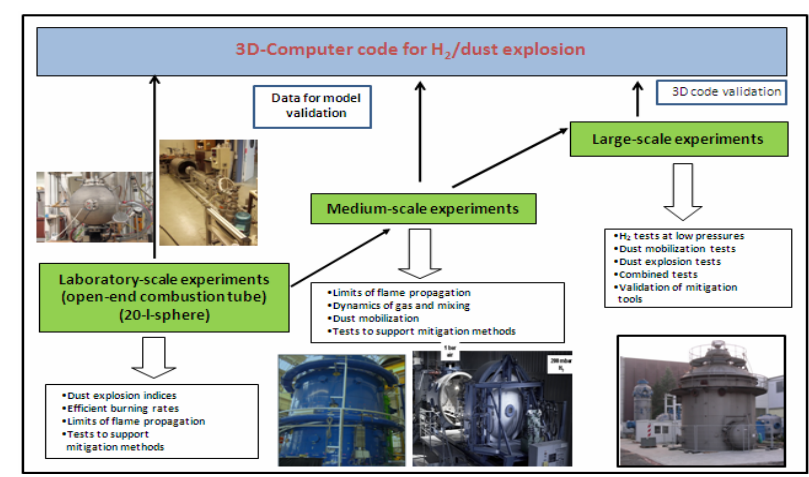

Fig. 5: Roadmap to H2/dust explosion modelling

\subsubsection{Tasks for numerical tool development}

In view of assessing and improving dust resuspension and mobilisation modeling (in particular for LOVA conditions), three different numerical benchmarks are on-going with two Computational Fluid Dynamic (CFD) codes (FLUENT-ENEA and GASFLOW-KIT) and a Monte Carlo code (DSMC-CEA). Particular attention is paid on the determination of the local gas velocity field for very low pressure conditions (100-1000 Pa) and on the turbulence models. One of the main tasks consists of defining the necessary additional experimental programme for full model validation.

The non reactive flow is calculated for a given accidental sequence and for a realistic geometry (with respect to ITER) with the CFD code GASFLOW (or CAST3MCEA but without dust). The 3D mixture composition is extracted and used by a CFD combustion code (COM3D or DET3D-KIT) allowing determination of 3D thermal and pressure loads on the VV (see fig. 6)

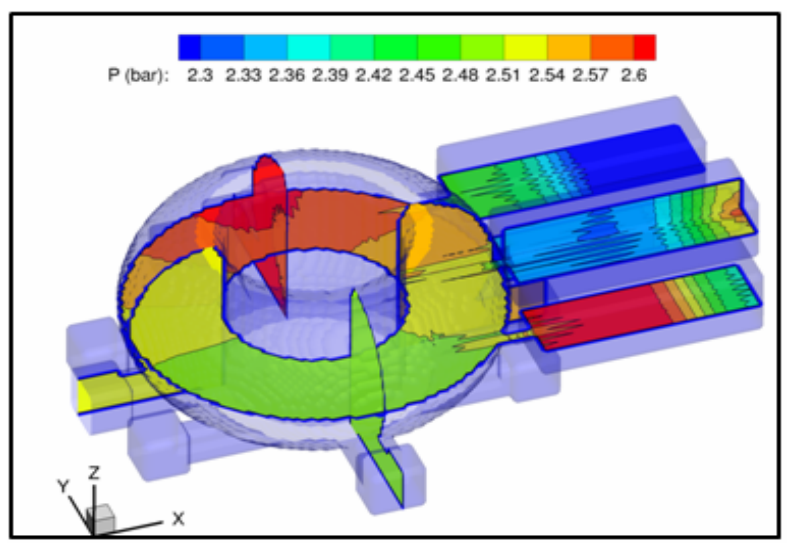

Fig.6: Exemple of pressure contours in the VV (COM3D)

Finally a 1D mechanical code (elasto-plastic deformation) is being developed and tested to evaluate the impact on the VV of the pressure and thermal loads calculated by the combustion code.

\subsubsection{Experimental tasks}

Small scale (separate effects) and medium scale experiments were and are being performed to provide relevant data for physics models validation and a final experimental programme on a large scale facility (integral experiment) is under definition in order to assess and validate the numerical tool on a relevant geometry and on relevant conditions with respect to ITER.

Experiments performed in a 20 liter sphere (DUSTEX facility-KIT) allowed determination of main explosion indices of dust-air and hybrid dust/H2-air mixture such as: maximal pressure, pressure rise rate, Limiting Oxygen Concentration (LOC) and energy of ignition. In particular it was shown that: 1) a strong energy source (chemical, a few tens of Joules) is necessary to trigger a dust-air cloud explosion (for W or C) 2) a H2 combustion (triggered by a weak ignition source-electric spark of a few $\mathrm{mJ}$ ) can trigger a dust-air cloud explosion 3 ) the maximum pressure is higher and the explosion occurs faster in the case of a mixture (H2/dust/air) 4) the LOC is lower for $\mathrm{W}$ dust than for $\mathrm{C}$ dust (7\% compare to 9\%).

The model implemented in DET3D for H2/dust/air explosion provides valuable results when compared to experiments (see fig. 7).

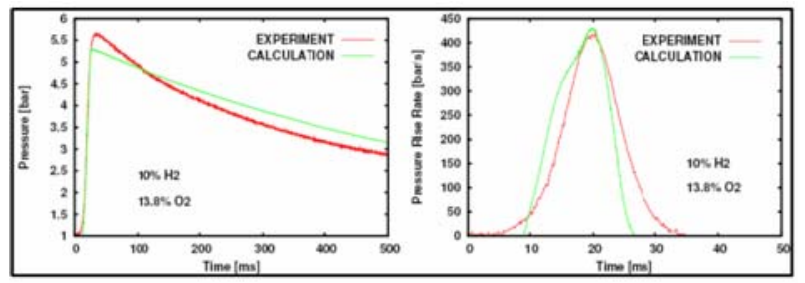

Fig. 7: experimental and computed pressure history and pressure rise rate for DUSTEX experiment with $1000 \mathrm{~g} / \mathrm{m}^{3} \mathrm{~W}$ dust

Further experiments, performed on long tubes (PROFLAM1\&2-KIT) equipped with row of thermocouples and pressure sensors allow determination of flame velocity. On-going efforts on flame velocity modeling are promising (see fig.8).

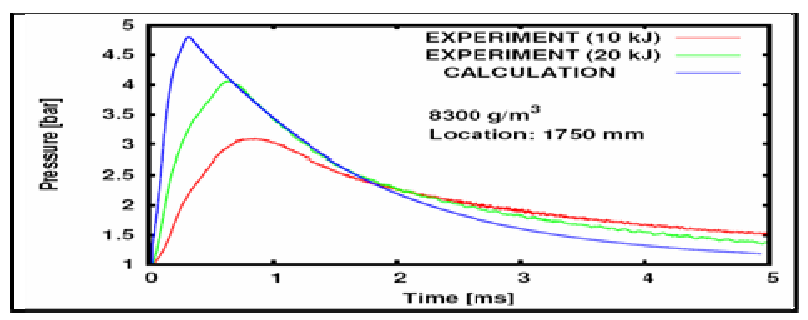

Fig. 8: Comparison of experimental and calculated pressure histories at $1750 \mathrm{~mm}$ from ignition

Furthermore, experimental campaigns have been performed on the MISTRA facility (CEA) in order to provide accurate data on gas mixing in realistic conditions. A benchmark based on MISTRA results is on-going with CAST3M and GASFLOW.

\section{References}

[1] Preliminary Safety Analysis of ITER, N. Taylor et al., Fusion Science and Technology, 56, 2009, 573-580Neill papers?

[2] Up-dated Modeling of Postulated Accidents Scenarios in ITER, S. Reyes et al, Fusion Science and Technology, vol. 56, nº2, pp. 789-793, 2009

[3] Recent Analysis of Key Plasma Wall Interactions Issues 
for ITER, J. Roth et al, PSI 18 Toledo, May 2008.

[4] On the use of a capacitive diaphragm gauge for dust detection in next-step fusion devices, G. Counsell et al, Review of Scientific Instruments, Volume 77, Issue 9, pp. 093501-093501-6 (2006).

[5] Dust in cavity reactivity measurements, D.A. Davydov et al, ITER Final report for TA G 81 TT 09 FR (T535), July 2001

[6] Consequences of Deuterium Retention and Release from Be-containing Mixed Materials for ITER Tritium Inventory Control, K. Sugiyama et al, J. Nuclear Material, accepted for publication 